\title{
Longstanding Persistent Atrial Fibrillation Ablation: How Do You Perform It?
}

Carola Gianni ${ }^{1, *}$, Andrea Natale ${ }^{1-4}$, Amin Al-Ahmad ${ }^{1}$

\section{ORCID IDs}

Gianni C (1) https://orcid.org/0000-0002-8174-7207

Natale A (1) https://orcid.org/0000-0002-5487-0728

Al-Ahmad A (1) https://orcid.org/0000-0002-8547-025X

\begin{abstract}
Longstanding-persistent atrial fibrillation is one of the most challenging arrhythmias to treat. While radiofrequency catheter ablation is highly effective in paroxysmal atrial fibrillation, pulmonary vein antral isolation (including posterior wall isolation) alone is not enough for nonparoxysmal atrial fibrillation, other targets should be sought in this population. In this case report, we will describe our approach in a typical patient presenting for a first-time ablation procedure for longstanding persistent atrial fibrillation.
\end{abstract}

KEYWORDS: Atrial fibrillation; Radiofrequency ablation; Catheter ablation. 


\section{INTRODUCTION}

Longstanding persistent atrial fibrillation (LSPAF) is one of the most challenging arrhythmias to treat. While radiofrequency (RF) catheter ablation is highly effective in paroxysmal atrial fibrillation (AF), pulmonary vein antral isolation (PVAI; including posterior wall - $\mathrm{PW}$ - isolation) alone is not enough for nonparoxysmal AF, other targets should be sought in this population. When AF persists, electrophysiological and structural changes of the atrial myocardium predispose to the development of triggers originating from sites other than the PVs (the so-called non-PV triggers) and make the atria the perfect substrate to sustain AF. To tackle the latter, several substrate-based ablation strategies with have been described for catheter ablation of LSPAF, including linear lesions to compartmentalize the left atrium (LA), to ablation of complex fractionated electrograms or autonomic ganglionated plexi, scar homogenization, or ablation of localized organized reentrant activity (drivers). However, none of them has been proven to be superior to PVAI alone ${ }^{1}$.

Our ablation strategy, on the other hand, is to target triggers: While substrate determines the persistence of AF, there is no AF without a trigger initiating it. In LSPAF, there are many common non-PV triggers that can be targeted empirically (such as coronary sinus - CS, left atrial appendage - LAA, or superior vena cava - SVC), but it is also important to actively sought atypical triggers by means of high-dose isoproterenol challenge. In the following case report, we will describe our approach in a typical patient presenting for a first-time ablation procedure for LSPAF.

\section{CASE REPORT AND DISCUSSION}

This is the case of a 74-year-old woman with a history of symptomatic LSPAF, initially diagnosed four years prior and refractory to both cardioversions (two prior failed attempts) and antiarrhythmic drugs (AADs). At the time of the initial evaluation, she was in poor rate control despite beta-blockers and digoxin, and was orally anticoagulated with apixaban. Her past medical history is significant for hypertension, which has been controlled for years with a combination of angiotensin-converting enzyme (ACE)-inhibitor and thiazide therapy. Her most recent echocardiographic evaluation showed a mix of hypertensive and arrhythmia-related heart disease with mild left ventricular (LV) concentric hypertrophy and LV ejection fraction reduction (45 to 50\%), severe biatrial enlargement (anteroposterior LA diameter $52 \mathrm{~mm}$ ), and functional mild to moderate mitral and tricuspid regurgitation. Given the failed attempts at pharmacological and electrical cardioversion, as well inadequate rate control and initial LV dysfunction, a decision was made to proceed with RF catheter ablation.

The ablation procedure is performed under general anesthesia, which is important because it improves catheter stability by means of controlling the patient's respiration and movement ${ }^{2}$. In addition, oral anticoagulation is uninterrupted (i.e. last dose of apixaban taken the morning of the procedure), with an adequate washout from any AADs, including digoxin and beta-blockers. The former minimizes the risk of periprocedural strokes while the latter reduce the chance of intraprocedural arrhythmias noninducibility. A urinary catheter is usually not necessary, especially with newer irrigated ablation catheters (flow of $\sim 15 \mathrm{~mL} / \mathrm{min}$ during RF) and use of vascular closure devices to promote early ambulation. Finally, a sensitive (i.e. responding fast to any change in intraluminal temperature) esophageal probe is positioned in esophagus, and moved close to the tip of the ablation catheter whenever ablating along the posterior aspect of the $\mathrm{LA}^{3}$.

After obtaining ultrasound-guided central venous access (two 8-Fr sheaths in the right femoral vein, one 11-Fr sheath in the left femoral vein, one 7-Fr sheath in the right internal jugular vein), a duodecapolar mapping catheter is positioned to record electrograms in the coronary sinus (CS) and crista terminalis. Dual transseptal access is obtained under intracardiac echocardiography (ICE)-guidance using a blunt RF transseptal needle, which we prefer because it requires minimal force to get across the septum, thus minimizing the risk of perforation. For mapping and ablation of the LA, we use a fixedcurve circular mapping catheter through a LAMP 90 transseptal sheath, and an irrigated-tip ablation catheter through a SL0 transseptal sheath. 
Electroanatomical voltage mapping (performed during AF) showed severe scarring, with silent PVs (Video 1; Fig. 1). In this cohort of patients (elderly female with LSPAF and severe scarring/silent PVs) the prevalence of non-PV triggers is high and, as such, in addition to PW isolation, we aim to perform empirical ablation of the LA septum, roof, lateral wall as empirical isolation of the LAA and CS. For ablation, we employ a high-power (at least $40 \mathrm{~W}$ ) short-duration strategy with a target contact force of 7-10 $\mathrm{g}$ and varying RF times based on both myocardial thickness and safety (longer in the anterior aspect of the LA, shorter for PW/veins). More specifically, while esophageal temperature readings are important and the catheter should be moved whenever an increase in temperature is noted, the probe is smaller than the esophagus. As such, to minimize the risk of esophageal thermal injury and potential for atrioesophageal fistula, it is important to move fast whenever ablating the posterior heart regardless of potential abatement.

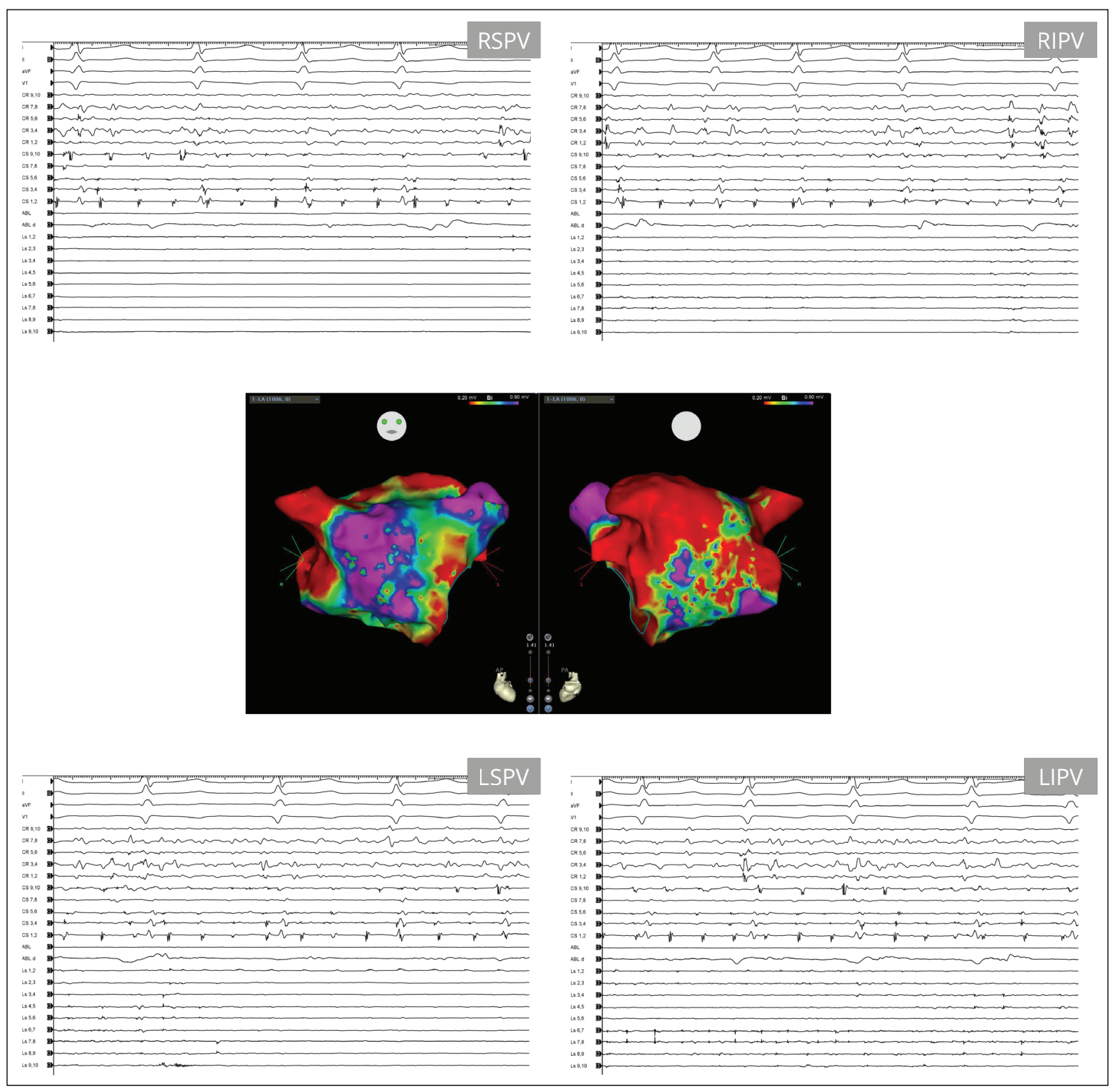

Figure 1. Electroanatomical voltage map and PV electrograms (Ls) showing severe scarring along with (almost) silent PVs. 
Most of LA ablation can be performed in AF, except for proximal CS and LAA isolation, which are best done in sinus rhythm to minimize the risk of atrioventricular block and facilitate LAA isolation, respectively. Indeed, most of the electrically active LA was ablated before external electrical cardioversion (Fig. 2), which was needed to complete LAA isolation. During sinus rhythm, it is easier to identify and target the areas of early activation, resulting in progressive delay up to isolation (Fig. 3). Whenever ablating around the LAA, it is important to closely monitor both contact force (it should be relatively stable, with values rarely exceeding $10 \mathrm{~g}$ ) and impedance (a sudden increase might point to ablation inside a pouch, with an increased risk of steam pop and perforation).

After external electrical cardioversion, the patient developed a spontaneous sustained focal atrial tachycardia originating from the interatrial septum, as evidenced by a reduction of the interval between crista terminalis and CS when compared to the corresponding sinus rhythm activation sequence (Fig. 4). This tachycardia was successfully mapped and ablated in the mid-anterior, superior aspect of the LA septum. At this point, the patient underwent drug challenge with high-dose isoproterenol, which elicited two typical non-PV triggers (Fig. 5), with tachycardias seen from the proximal CS (which was not yet abled) and the LAA (which displayed partial antral reconnection). Reisolation of the LAA addressed the latter, whereas the proximal CS tachycardia terminated while ablating the endocardial aspect of the CS, within the LA. The CS was finally completely isolated by means of epicardial (within the CS) ablation. As stated, ablation of the proximal CS should be performed in sinus rhythm to monitor the PR interval and reduce the risk of atrioventricular block. Before the end of the procedure, the circular mapping catheter positioned in the SVC, showed a silent vein, which was therefore not targeted for ablation.

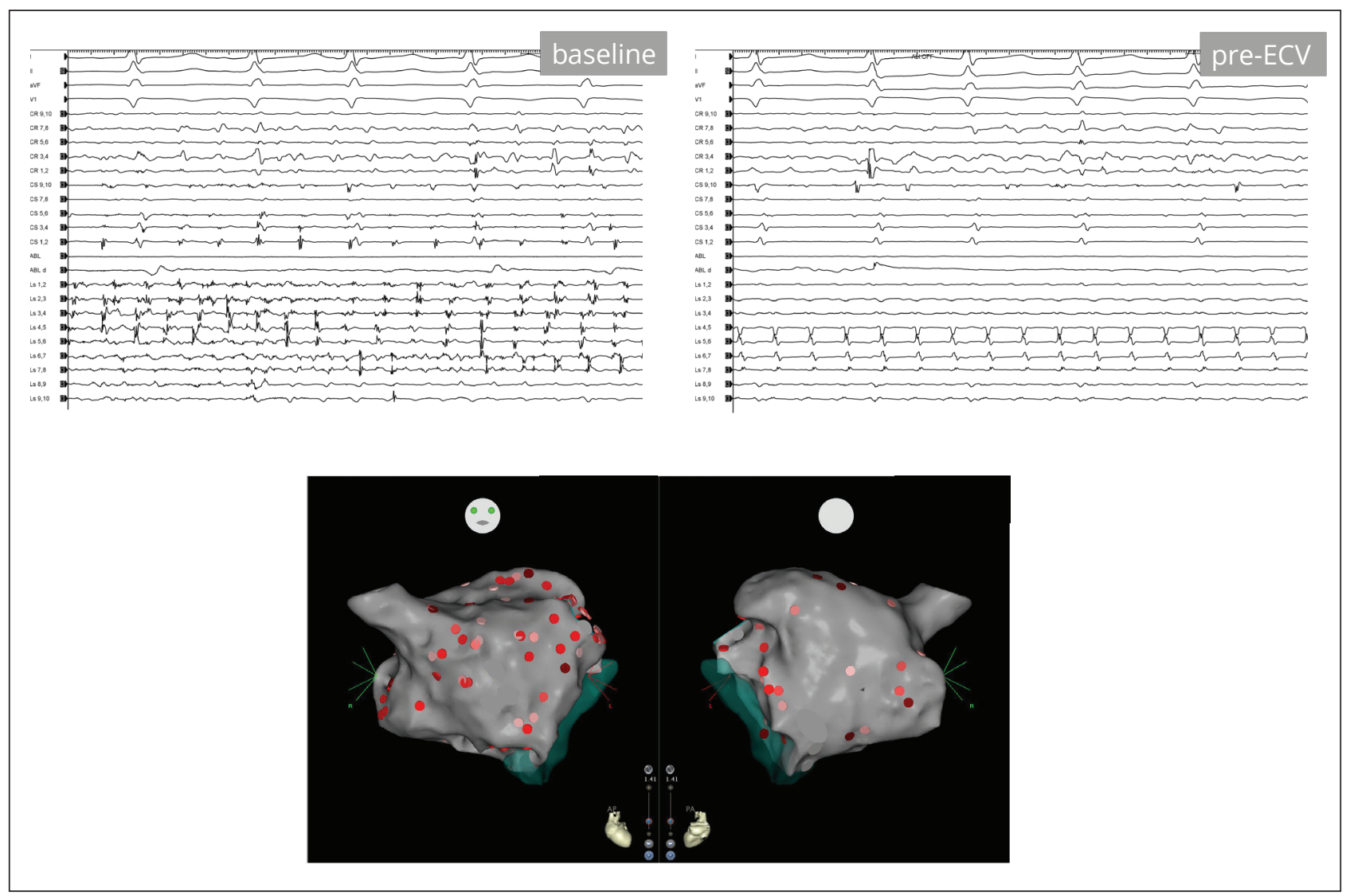

Figure 2. Intracardiac electrograms (top) showing the CS and LAA (Ls) at baseline and before external electrical cardioversion (ECV). As the lesion set (bottom) shows, most of the LA was ablated before ECV. 


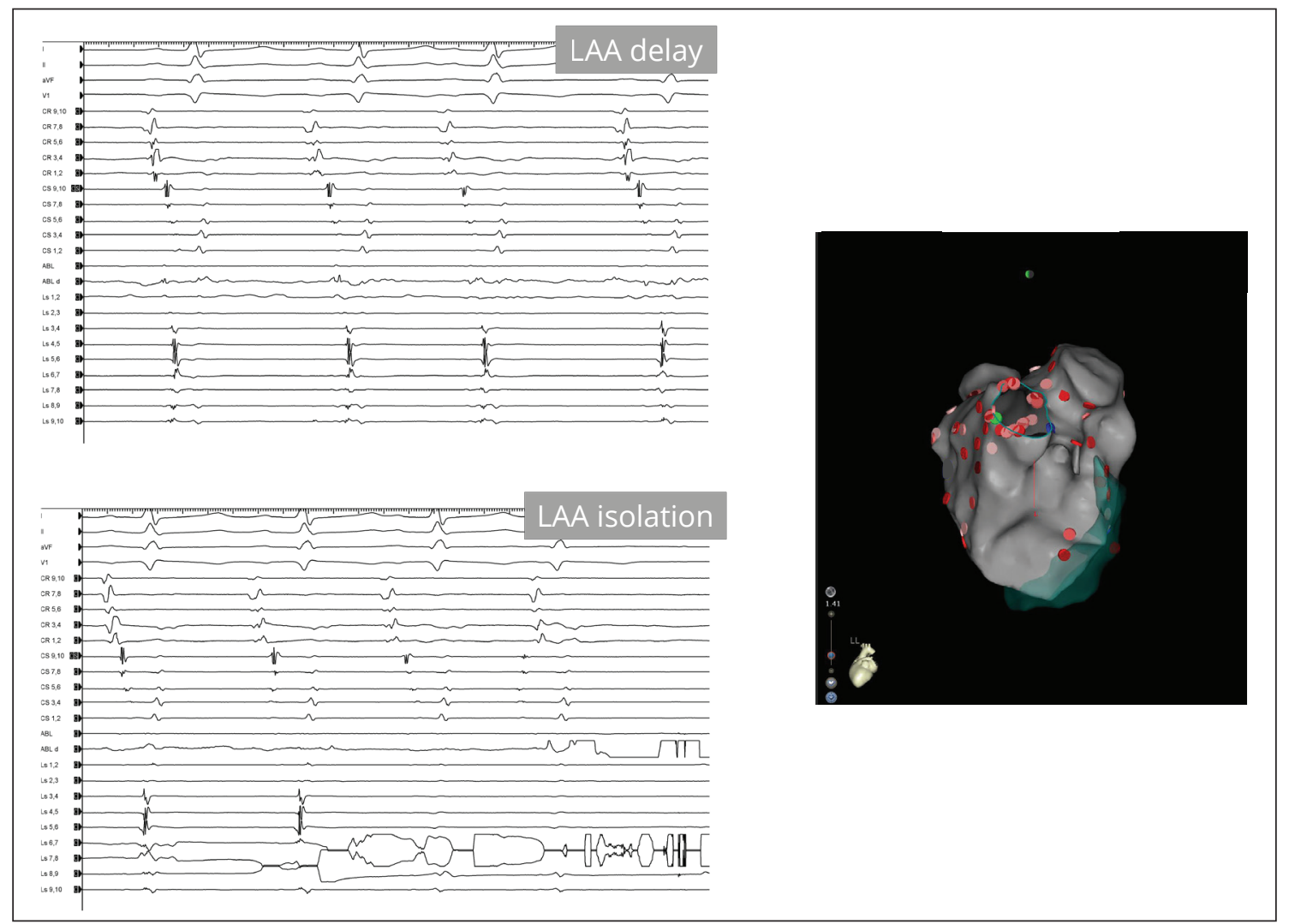

Figure 3. Left atrial appendage delay (blue dot) and isolation (green dot), performed in sinus rhythm.

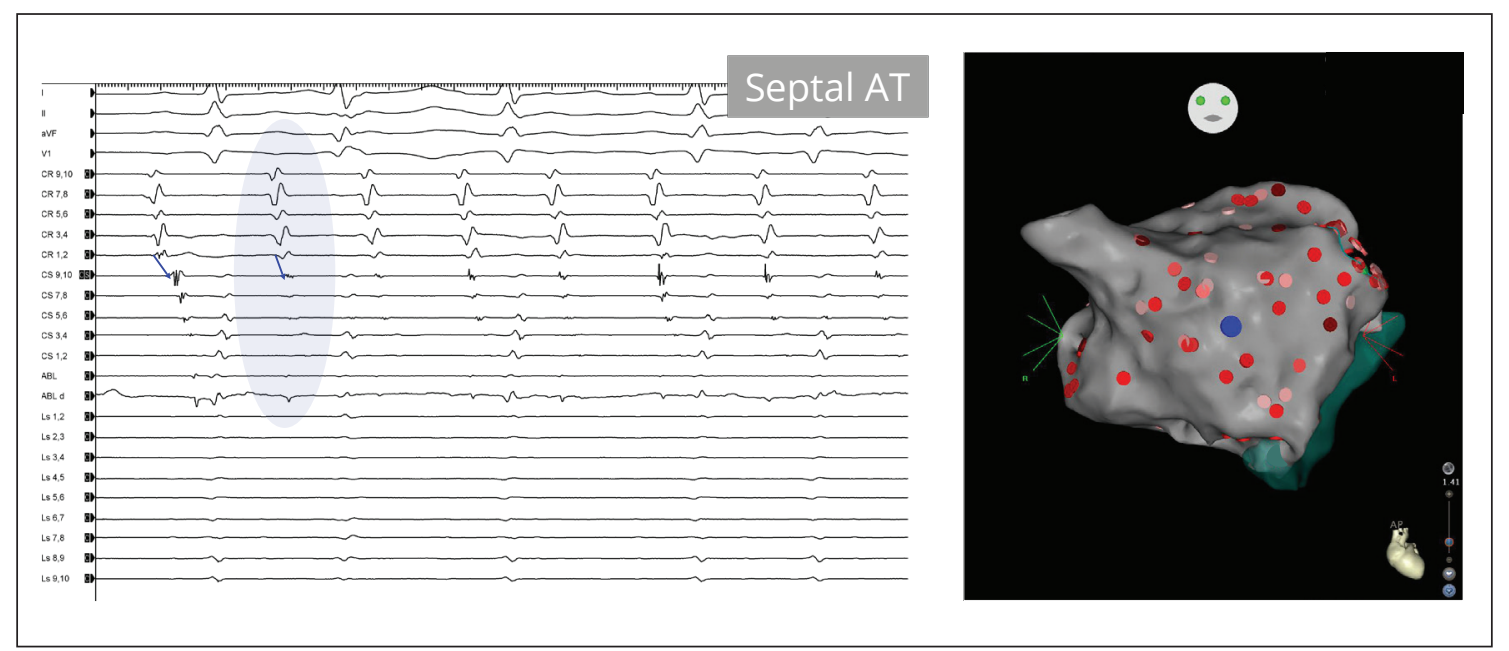

Figure 4. Spontaneous septal atrial tachycardia, which is easily identified by observing reduction of the interval between distal crista terminalis and proximal CS compared to that in sinus rhythm. Blue dot on the mid-anterior, superior aspect of the septum indicates the termination spot. 


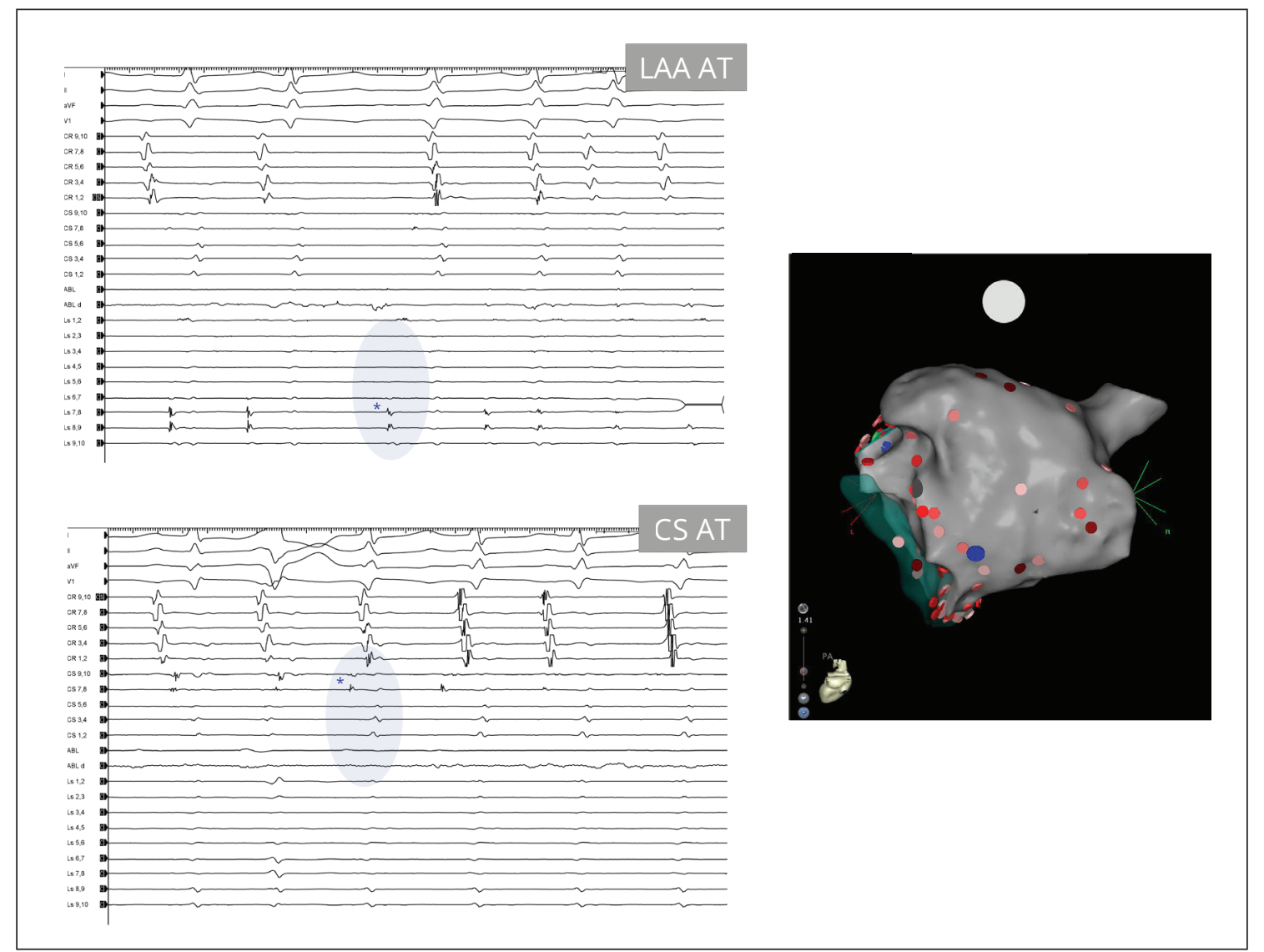

Figure 5. High-dose isoproterenol induced LAA and proximal CS tachycardias. Blue dot on the floor of the LA (across the proximal CS) is the termination spot of the CS tachycardia, whereas LAA tachycardia terminated with its reisolation.

The patient was discharged with AADs, as it is our protocol for the 2-month blanking period following extensive ablation. Despite AADs, the patient developed recurrent symptomatic atypical AFL and underwent a repeat RF ablation about 6 months after the first procedure. Mapping of the LA demonstrated reconnection of the proximal CS and LAA, as well as areas of surviving myocardium along the LA roof. With RF, reconnection of previously isolated structures is not uncommon, and a second procedure might be necessary to achieve chronic CS/LAA isolation and improve long-term outcomes. Both CS and LAA were reisolated, and the LA roof was ablated to obtain electrical silence. Of note, we did not attempt to induce the clinical arrhythmia. Indeed, there is no correlation between procedural AF/ AFL termination and arrhythmia recurrence in nonparoxysmal AF patients: despite effective substrate modification, without targeting non-PV sites, outcomes do not improve ${ }^{4,5}$. This can be explained on a mechanistic point of view: while the substrate determines the persistence of AF/AFL, there is no AF/AFL without a trigger initiating it.

Following the second procedure, the patient did well on an arrhythmia standpoint and her AADs were finally discontinued with no evidence of recurrence, as evidenced by absence of symptoms as well as no arrhythmias detected with routine ECG monitoring. Conversely, OAC was continued indefinitely given persistent LAA isolation.

\section{AUTHOR'S CONTRIBUTION}

Conceptualization, Gianni C., Natale A. and Al-Ahmad A.; Methodology, Gianni C., Natale A. and Al-Ahmad A.; Investigation, Gianni C., Natale A. and Al-Ahmad A.; Writing - Original Draft, Gianni C., Natale A. and Al-Ahmad A.; Writing - Review and Editing, Gianni C., Natale A. and Al-Ahmad A.; Resources, Gianni C., Natale A. and Al-Ahmad A.; Supervision, Gianni C., Natale A. and Al-Ahmad A. 


\section{REFERENCES}

1. Gianni C, Mohanty S, Trivedi C, Di Biase L, Natale A. Novel concepts and approaches in ablation of atrial fibrillation: the role of nonpulmonary vein triggers. EP Eur. 2018;20(10):1566-76. https://doi.org/10.1093/europace/euy034

2. Di Biase L, Conti S, Mohanty P, Bai R, Sanchez J, Walton D, et al. General anesthesia reduces the prevalence of pulmonary vein reconnection during repeat ablation when compared with conscious sedation: Results from a randomized study. Heart Rhythm. 2011;8(3):368-72. https://doi.org/10.1016/j.hrthm.2010.10.043

3. Gianni C, Atoui M, Mohanty S, Trivedi C, Bai R, Al-Ahmad A, et al. Difference in thermodynamics between two types of esophageal temperature probes: Insights from an experimental study. Heart Rhythm. 2016;13(11):2195-200. https://doi.org/10.1016/j. hrthm.2016.07.021

4. O'Neill MD, Wright M, Knecht S, Jaïs P, Hocini M, Takahashi $Y$, Jönsson A, et al. Long-term follow-up of persistent atrial fibrillation ablation using termination as a procedural endpoint. Eur Heart J. 2009;30(9):1105-12. https://doi.org/10.1093/eurheartj/ehp063

5. Elayi CS, Di Biase L, Barrett C, Ching CK, al Aly M, Lucciola M, et al. Atrial fibrillation termination as a procedural endpoint during ablation in long-standing persistent atrial fibrillation. Heart Rhythm. 2010;7(9):1216-23. https://doi.org/10.1016/j.hrthm.2010.01.038 